\title{
Endoscopic Ultrasound-Guided Diagnosis of Pancreatic Cystic Lesions: Microforceps or Molecular Markers!
}

Pancreatic cystic lesions (PCLs), especially asymptomatic, are being increasingly recognized in recent years due to the frequent use of cross-sectional imaging techniques for various abdominal indications. ${ }^{[1]}$ The PCLs can be broadly differentiated into mucinous cysts that comprises mucinous cystic neoplasm (MCN), intraductal papillary mucinous neoplasm (IPMN)), and nonmucinous cysts that includes serous cystadenoma (SCN), solid pseudopapillary neoplasm (SPEN) and pseudocyst. All PCLs are not malignant with pseudocyst and SCN being benign and MCN, IPMN, and SPEN having a malignant potential with capacity to transformed into invasive cancer. ${ }^{[2,3]}$ The challenge before the clinician is to differentiate the mucinous from nonmucinous cysts and also characterize the mucinous cysts as benign, malignant, and potentially malignant. Endoscopic ultrasound (EUS) has dramatically improved our ability to achieve this goal by improved morphological characterization of the cysts as well as safer and accurate sampling of these cysts. In morphological characterization, EUS provides not only detailed information about the morphological features such as size and location of the cyst, pancreatic duct dilatation and its relationship with the cyst, presence or absence of mural nodule, and regional lymphadenopathy. EUS-guided sampling of the cyst provides the fluid for the cytological and molecular analysis. ${ }^{[4,5]}$ However, despite these advances, we have not been able to achieve high diagnostic accuracy for the diagnosis of PCLs. ${ }^{[6-8]}$ The cytological evaluation of cyst fluid has a low sensitivity and specificity due to scant cellularity and interpretation challenges due to gastrointestinal tract contaminates as well as the presence of degenerative changes in cells. Therefore, majority of pancreatic cyst are either diagnosed at advanced stages or are not accurately differentiated on radiology as well as cytology resulting in diagnostic dilemma. There has been constant endeavor to improve on the diagnostic capabilities of EUS- and EUS-guided fine-needle aspiration (FNA) for accurately characterizing the PCLs. Recently, a newly designed EUS-guided micro biopsy forceps has been introduced in the hope of getting better tissue from the cyst wall for pathological diagnosis. In addition, newer molecular markers and cytological features are being evaluated in cyst fluid to enhance the diagnostic accuracy of EUS FNA. ${ }^{[9-12]}$ In this news and views, we will discuss three studies on these newer developments in EUS-guided evaluation of the PCLs. ${ }^{[9-11]}$
In a multicenter retrospective study done by Basar et al. between 2015 and 2016, newly designed single-use Moray microforceps biopsy (MFB) device (U. S. Endoscopy, Mentor, Ohio) was used along with a 19 G EUS FNA needle for acquisition of cyst wall for histopathology. ${ }^{[10]}$ After performing a detailed EUS that included the number as well as location of PCLs, size, presence or absence of the septations, presence or absence of adjacent mass, and mural nodules, the cyst was punctured using 19G flex FNA needle (Boston Scientific, Marlborough, MA, USA). The cyst fluid was aspirated, and thereafter, without removing the needle, the MFB device was inserted through the needle and pinch biopsy was taken from cyst wall, septations, mural nodules, and from adjacent masses if present. The biopsy was obtained in the order of adjacent mass first followed by mural nodule, cyst wall, and septations, respectively. If biopsy specimen were thought of being insufficient, repeat passes were made. Aspirated cyst fluid was sent for carcinoembryonic antigen (CEA) and cytological analysis, and MFB samples were sent in formalin solution and processed as routine histology specimens as processed.

The authors used the term "tissue acquisition yield" to define the ability to collect fluid or tissue for analysis and it was calculated as the number of patients with fluid or tissue obtained by aspiration or MFB, which was either diagnostic or nondiagnostic, divided by the total number of patients. The diagnostic yield was evaluated at three different levels, with level 1 analysis being defined as the ability to differentiate between mucinous and nonmucinous cyst, level 2 analysis being defined as ability to detect a cyst at high risk for malignancy, and level 3 analysis being defined as the ability to make specific diagnosis such as IPMN, MCN, SCN, and pseudocyst).

The cyst was defined as mucinous if there was the presence of extracellular mucin or mucinous epithelial on cytology and all other cysts were diagnosed as nonmucinous. Also, cyst fluid CEA >192 ng/ml was considered as diagnostic for mucinous cyst. Cytology was considered as high risk if there was a presence of malignant cells or cyst with high-grade epithelial dysplasia or cystic neuroendocrine tumor. Histological evaluation of MFB tissue followed the standard histological definitions. In mucinous cyst, histology was diagnostic as MCN if there was the presence of subepithelial ovarian stroma and its absence diagnosed IPMN. 
Forty-two patients (23 females and 19 males) with mean age 69 years (range 27-91 years) underwent EUS-FNA and MFB procedure. In $16(38.5 \%)$ patients, the cyst was located in head of pancreas, in 17 patients $(40.5 \%)$ body, and in $9(21.4 \%)$ in tail of the pancreas. The mean size of cyst was $28.2 \mathrm{~mm}$ (range 12-60 mm), and in 25 patients, the cysts were multiseptated and in 17 was unilocular. The mural nodule was present in four patients and adjacent mass in one patient. The cyst fluid was obtained in 37 patients and yield of EUS FNA acquisition of fluid was $88.1 \%$, and MBF tissue acquisition yield was $90.4 \%$. The pinch biopsy was not sufficient in 4 patients $(9.6 \%)$. In 30 patients $(71.4 \%)$, MBF biopsy was obtained from cyst wall, in 6 patients (14.4\%) MBF were taken from septations, and in one patient $(2.4 \%)$ from the adjacent mass. The yield of MBF tissue biopsy was not significant in groups of size or location of cyst as well as presence or absence of septations. During the procedure or before discharge, no serious complications were observed except one patient had self-limiting mild pain abdomen, and one patient had self-limiting intracystic bleed.

In Level 1 analysis (ability to distinguish mucinous from to non-mucinous cyst), the diagnostic yield of cyst fluid cytology was similar to that of MBF histology (47.6\% vs. $61.9 \%$, patients; $P=188)$. Excluding acquisition failure, $45.9 \%$ and $31.5 \%$ patients were non-diagnostic by cyst fluid cytology and MBF histology, respectively. The mean CEA was $863 \mathrm{ng} / \mathrm{ml}$ in mucinous cyst and $47.4 \mathrm{ng} / \mathrm{ml}$ in nonmucinous cyst $(P=003)$. For Level II analysis (risk of malignancy), the diagnostic yield of cyst fluid cytology was similar to that of MBF histology (54.7\% vs. $71.5 \%$, patients; $P=113$ ). However, there was a significant difference in the Level III diagnostic tissue yield between cyst fluid cytology and MFB histology (4.8\% and $35.7 \%$, respectively; $P=001$, respectively). The authors concluded that the cyst tissue acquisition yield for MFBs was 90\% and although the diagnostic yield of cytology of cyst fluid and MFB were comparable in distinguishing mucinous and nonmucinous cysts as well as detecting cysts at high risk for malignancy, MFB was superior to cytology for providing a specific cyst diagnosis.

In an interesting observation, Chebib et al. ${ }^{[11]}$ reported the utility of detecting desiccated appearing or inspissated cyst contents in patients with neoplastic mucinous cysts. This inspissated material has distinctly fibrillary, rounded, or granular architecture that is different from thin mucin or cellular debris. The authors retrospectively studied the case records of pancreatic cysts containing inspissated cyst contents on EUS-FNA between 1996 and 2015. Cytology slides were prepared by either direct smear, liquid preparation, or cytospin preparation method.

In this study, authors reviewed 1456 pancreatic cysts, and 28 (8 males) of these patients had inspissated cyst fluid. The average size of the cyst was $2.2 \mathrm{~cm}$ (range from 0.9 to $8.0 \mathrm{~cm}$ ), and the cyst was located in head, body, and tail in 10,8 , and 9 , patients, respectively. In one patient, the site of the cyst was not mentioned. The diagnosis was made on the basis of histology in 7 patients (4 patients diagnosed as MCN, 2 were lymphoepithelial cysts, and 1 was diagnosed as chronic pancreatitis) and the combination of cytology/cyst fluid analysis and molecular testing of KRAS/GNAS in 17 cysts. The cytomorphology of the inspissated pancreatic cyst fluid showed $15(54 \%)$ cases with very similar fibrillary, fan-like structures that were typically blue to purple color on Papanicolaou stain and ranged from fibrillary fan-like structure (54\%), ball-like structures (57\%), and granular material (43\%). The fibrillary inspissated material was seen in $11(73 \%)$ neoplastic mucinous cysts and 2 pseudocysts, $1 \mathrm{SCN}$, and 1 cyst of uncertain type. The presence of fibrillary inspissated cyst material on cytology was found to be having a positive predictive value of $79 \%$ and a specificity of $63 \%$ for a neoplastic mucinous cyst. The authors concluded that the presence of inspissated cyst fluid with fibrillary architecture is not highly specific for mucinous cysts but should be recognized by cytopathologists and interpreted as at least "atypical."

Faias et al., in a retrospective cohort study, evaluated the role of KRAS and GNAS mutational status in pancreatic cysts in identifying mucinous cysts, particularly high-risk/malignant lesions, and to evaluate its added value in decision making. ${ }^{[9]}$ Fifty-two frozen samples of pancreatic cystic fluid obtained by EUS-FNA between 2008 and 2014 were analyzed. The EUS still images of these 52 patients $(67 \%$ females, with a mean age of $59 \pm 15$ years) were reviewed for morphological features such as size, septations, mural nodules, wall thickening, mass, main pancreatic duct features, and its communication with cyst. The mutations of GNAS (exons 8 and 9) and KRAS (exons 2 and 3) genes were analyzed using Sanger sequencing.

The mean size of the cyst was $3.9 \mathrm{~cm}$ with the predominant location being head and body of pancreas. Nearly $75 \%$ cysts were incidental and asymptomatic whereas $23 \%$ patients presented with abdominal pain. After clinical, radiological, and cyst fluid analysis, cysts were classified as mucinous in 21 patients $(40 \%)$ (14 low risks and seven malignant) and nonmucinous in 31 patients $(60 \%)$. After EUS-FNA, 11 patients underwent surgery, six were given chemotherapy or 
palliation, one had endoscopic drainage, and 34 are on follow-up (mean of 57 months). KRAS mutation was detected in nine and GNAS in two samples, and their detection did not change the clinical decision obtained after clinical, radiological, and cyst fluid analysis. Patients with KRAS mutations were older $(P=0.01)$ with cysts being more commonly mucinous $(P=0.001)$ and malignant $(P=0.01)$. KRAS mutations were present in both low-risk $(n=4)$ and malignant mucinous lesions $(n=5)$. For identifying mucinous lesions, CEA $>192 \mathrm{ng} / \mathrm{mL}$ in the cyst fluid was the best test (Area under curve [AUC] receiver operating characteristics $[\mathrm{ROC}]=93 \%$ ), and molecular analysis did not perform as well $(\mathrm{AUC}=0.72)$, with a low sensitivity of $50 \%$.In addition, for identifying malignant/high-risk mucinous lesions, EUS imaging had the best accuracy (AUC ROC $=88 \%$ ). After molecular analysis, a modification in cyst classification occurred in ten patients, but was correct in only two, a pseudocyst reclassified as IPMN and a malignant cyst as a nonmucinous cyst. The authors concluded that in their cohort of patients with PCL, KRAS, and GNAS mutations had no significant diagnostic benefit in comparison with conventional testing.

\section{Commentary}

Although EUS and FNA are commonly done in patients with PCLs with an objective of determining mucinous cysts and differentiating malignant from nonmalignant cysts and despite its advantage of enhanced imaging with better resolution, EUS still has not been able to achieve these objectives effectively. The low diagnostic yield of cytology and lack of satisfactory sensitivity and specificity of cystic fluid markers have led on to the development of new devices as well as molecular technologies trying to overcome this problems. ${ }^{[12]}$ The newer devices include the cytology brush (Echobrush), the small mini-biopsy forceps, the cystoscopy, and the needle confocal endomicroscopy. The newer molecular markers include identifying mutations in DNA of particular genes as KRAS, GNAS, VHL, CDKN2A, and other genes in the cystic fluid. The study by Basar et al. has shown that although EUS-FNA cyst fluid analysis has similar value to MFB in differentiation between mucinous/non-mucinous cysts and accurately diagnosing high-risk cysts, it cannot usually accurately diagnose the type of cyst. ${ }^{[10]}$ As MFB provides tissue from the cyst wall, septations, and mural nodules for histology, it can help in accurately diagnosing the type of cyst and also provide providing tissue for ancillary studies such as immunohistochemistry. Moreover, the subepithelial cyst wall tissue can also be biopsied to show the ovarian type stroma and thus helping in differentiating an MCN from IPMN. The small sample size and retrospective study design were the major limitations of this study and a prospective multicenter study with more patients is needed to validate the reproducibility, safety, and usefulness of MFB for evaluating PCLs. Chebib et al. in a small sample size study reported that fanlike fibrillary structures that stain blue-purple to brown on Papanicolaou stain may be associated with a mucinous etiology with a positive predictive value of $79 \%{ }^{[10]}$ It is an interesting observation that may not dramatically change our practice but given their association with neoplastic mucinous cysts could help in appropriately diagnosing PCLs by combining this information with cytology, imaging, and molecular pathology information. Molecular analysis of cyst fluid is useful for PCLs evaluation; however, its performance in clinical practice remains unclear. Faias et al. also reported that KRAS mutations were highly specific to identify mucinous lesions with $100 \%$ specificity, but moderate discriminative power $(\mathrm{AUC}=0.72)$ and low sensitivity (50\%) and also detection of GNAS mutation did not improve diagnosis as only two samples had a GNAS mutation, and in both of those patients, a concomitant KRAS mutation was also present. ${ }^{[9]}$ In contrast to the expectations, the authors found that cyst fluid CEA level was more accurate for low-risk mucinous cysts and combined imaging and cytology was more accurate for high-risk mucinous/malignant cysts. Although more sensitive next-generation sequencing was not used in this study, it dispels the euphoria around the mutational analysis in PCLs. It seems that we need to study more novel mutations and also evaluate the use of mutational analysis to diagnose mucinous cysts in young patients who have nondiagnostic cytology and cyst fluid CEA $<192 \mathrm{ng} / \mathrm{mL}$.

Ravi Kumar Sharma, Surinder Singh Rana

Department of Gastroenterology, Postgraduate Institute of Medical Education and Research, Chandigarh, India

Address for correspondence: Dr. Surinder Singh Rana, Department of Gastroenterology, Postgraduate Institute of Medical Education and Research, Chandigarh - 160 012, India. E-mail: drsurinderrana@gmail.com

\section{REFERENCES}

1. Laffan TA, Horton KM, Klein AP, Berlanstein B, Siegelman SS, Kawamoto S, et al. Prevalence of unsuspected pancreatic cysts on MDCT. AJR Am J Roentgenol 2008;191:802-7.

2. Khalid A, Brugge W. ACG practice guidelines for the diagnosis and management of neoplastic pancreatic cysts. Am J Gastroenterol 2007;102:2339-49.

3. Hruban RH, Maitra A, Kern SE, Goggins M. Precursors to pancreatic cancer. Gastroenterol Clin North Am 2007;36:831-49, vi.

4. Mortelé KJ. Cystic pancreatic neoplasms: Imaging features and management strategy. Semin Roentgenol 2013;48:253-63. 
5. Brugge WR. The use of EUS to diagnose cystic neoplasms of the pancreas. Gastrointest Endosc 2009;69:S203-9.

6. Pitman MB, Genevay M, Yaeger K, Chebib I, Turner BG, Mino-Kenudson $\mathrm{M}$, et al. High-grade atypical epithelial cells in pancreatic mucinous cysts are a more accurate predictor of malignancy than "positive" cytology. Cancer Cytopathol 2010;118:434-40.

7. Springer S, Wang Y, Dal Molin M, Masica DL, Jiao Y, Kinde I, et al. A combination of molecular markers and clinical features improve the classification of pancreatic cysts. Gastroenterology 2015;149:1501-10.

8. Zhan XB, Wang B, Liu F, Ye XF, Jin ZD, Li ZS, et al. Cyst fluid carcinoembryonic antigen concentration and cytology by endosonography-guided fine needle aspiration in predicting malignant pancreatic mucinous cystic neoplasms. J Dig Dis 2013;14:191-5.

9. Faias S, Duarte M, Albuquerque C, da Silva JP, Fonseca R, Roque R, et al. Clinical impact of KRAS and GNAS analysis added to CEA and cytology in pancreatic cystic fluid obtained by EUS-FNA. Dig Dis Sci 2018;63:2351-61.

10. Basar O, Yuksel O, Yang DJ, Samarasena J, Forcione D, DiMaio CJ, et al. Feasibility and safety of microforceps biopsy in the diagnosis of pancreatic cysts. Gastrointest Endosc 2018;88:79-86.

11. Chebib I, Albanese E, Scourtas A, Pitman MB. Inspissated cyst fluid in endoscopic ultrasound-guided fine needle aspiration of pancreatic cysts. Diagn Cytopathol 2018;46:395-9.

12. Lariño-Noia J, Iglesias-Garcia $J$, de la Iglesia-Garcia $D$, Dominguez-Muñoz JE. EUS-FNA in cystic pancreatic lesions: Where are we now and where are we headed in the future? Endosc Ultrasound 2018;7:102-9.

This is an open access journal, and articles are distributed under the terms of the Creative Commons Attribution-NonCommercial-ShareAlike 4.0 License, which allows others to remix, tweak, and build upon the work non-commercially, as long as appropriate credit is given and the new creations are licensed under the identical terms.

\section{Access this article online}

$\begin{array}{ll}\text { Quick Response Code: } & \text { Website: www.jdeonline.in } \\ & \\ \end{array}$

How to cite this article: Sharma RK, Rana SS. Endoscopic ultrasound-guided diagnosis of pancreatic cystic lesions: Microforceps or molecular markers!. J Dig Endosc 2018;9:213-6. 